\section{El asalto a las relaciones pedagógicas. Educar en contextos de pandillas}

\section{Pauline Martin ${ }^{1}$ \\ Wim Savenije ${ }^{2}$}

\section{Resumen}

Las escuelas públicas salvadoreñas experimentan niveles preocupantes de inseguridad al interior y exterior de la institución, en relación con la presencia de pandillas (MINED, 2018a). Los esfuerzos de prevención de violencia visualizan a la escuela como un espacio que facilita el acceso a los jóvenes (Flores y Galdámez, 2021), pero poco se conoce sobre cómo la presencia de pandillas y estudiantes relacionados a ellas afecta el funcionamiento cotidiano de la escuela y la relación pedagógica en el aula. Este estudio, basado en seis estudios de caso realizados en el 2018 , identifica que la presencia de estos estudiantes genera temor en los docentes, erosiona la autoridad pedagógica y afecta la calidad de la educación.

\section{Introducción}

En El Salvador, la presencia de pandillas es un fenómeno que se hospeda en comunidades y colonias que sufren altos niveles de marginación y exclusión social, económica y política, lo cual se traduce en falta de oportunidades y acceso a servicios básicos; además, en una concomitante fragmentación social de los habitantes (Smutt y Miranda, 1998; Savenije \& Andrade-Eekhoff, 2003;

1 Directora de la Maestría en Política y Evaluación Educativa de la Universidad Centroamericana José Simeón Cañas; coordinadora e investigadora del programa de investigación "Educación en situaciones de riesgo y conflicto".

2 Doctor en Ciencias Sociales, investigador principal del programa de investigación "Educación en situaciones de riesgo y conflicto". 
Savenije, 2009). Las pandillas se apoderan de sus espacios inmediatos y los consideran como sus territorios, entrando en conflicto con los miembros de pandillas rivales que no respetan esa autoadjudicación. Proteger el barrio de otras pandillas se ha vuelto una de las principales razones de la violencia en las que incurren las pandillas (Baires et al., 2006). Este control territorial afecta a las escuelas, ya que el sistema educativo las ubica en zonas que entretejen con las áreas que las pandillas consideran parte de su territorio.

La presencia de jóvenes vinculados a las pandillas dentro de la escuela y en sus entornos representa un factor de riesgo para los centros educativos del país. En el 2018, 42.93 \% de las escuelas reportó que la presencia de pandillas en la comunidad representaba el mayor riesgo externo, y un $15.49 \%$ de las escuelas reportó la presencia de pandillas como factor de riesgo interno, bajando del $27.67 \%$ en el 2016 (MINED, 2018a). Estos datos reflejan que la escuela es percibida como un espacio relativamente seguro en la comunidad, aunque también genera preguntas sobre la influencia de pandillas al interior del centro educativo.

El funcionamiento de las escuelas públicas situadas en zonas con presencia de pandillas es el tema del programa de investigación "Educación en situaciones de riesgo y conflicto" de la Maestría en Política y Evaluación Educativa de la Universidad Centroamericana José Simeón Cañas de El Salvador, que investiga el fenómeno desde 2017. El presente artículo inquiere las dinámicas educativas entre docentes y estudiantes en dichas escuelas y pregunta cómo la presencia de pandillas en las aledañas afecta la relación pedagógica en el aula y el rol del docente. El objetivo es acercarse a la relación educativa siendo el núcleo de la labor del docente y fundamental en el proceso de aprendizaje-enseñanza. El artículo enfoca el aspecto relacional de la educación, más allá de los temas más habituales, como la asistencia de los estudiantes, la gobernanza de la escuela y la definición de un currículo u otros aspectos. El argumento se basa en los hallazgos de la segunda fase del programa de investigación que se implementó en 2018.

En el pasado, se vinculaba el abandono de los estudios con la afiliación a una pandilla. Por ejemplo, un estudio del Área Metropolitana de San Salvador (AMSS) reveló que, en las familias con hijos pertenecientes a una pandilla, solo el $55.3 \%$ tiene a sus hijos con edades entre los 10 y 18 años que asisten a la escuela; mientras que en las familias que no tienen hijos integrantes de las pandillas, el 95.3 \% estudia (Smutt y Miranda, 1998, p. 97). Aunque muchos de sus integrantes se rehusaban estudiar, las pandillas nunca abandonaron la escuela. Los centros educativos ubicados en zonas con presencia de pandillas son considerados parte integral de su territorio (Savenije \& Van der Borgh, 2015). La escuela pública es el lugar donde los jóvenes cercanos a las pandillas pasan parte de su tiempo; al mismo tiempo, estando allí, mantienen a la pandilla informada sobre lo que ocurre en la institución. La presencia de alumnos cercanos a las pandillas, o alumnos pandilleros, puede provocar tensiones con los docentes, disminuir la autoridad educativa de los profesores y convertir la escuela en un espacio inseguro (PNUD, 2013, pp. 221-223). No obstante, el centro educativo también puede ser un espacio apreciado por la pandilla, especialmente cuando los pandilleros tienen a sus hijos estudiando ahí y desean que reciban una buena educación (USAID-ECCN, 2016). Por otro lado, en El Salvador la escuela pública también es señalada como eje central de prevención (Consejo Nacional de Educación [CONED], 2016) y como un lugar donde se debe fomentar la cultura de paz y sana convivencia (MINED, 2018b). Muchos esfuerzos de prevención han enfocado tanto un abordaje de seguridad pública, como un enfoque educativo (Cuéllar-Marchelli y Góchez, 2017).

Existen pocos estudios sobre la influencia de la inseguridad en el funcionamiento de los centros educativos públicos y el trabajo docente en el aula. Es necesario comprender las situaciones que enfrentan los profesores 
para saber cómo responder y apoyarlos. Aunque las escuelas están operando con aparente normalidad, sus relaciones y su calidad de la educación pueden estar profundamente afectadas. Ante ello, el presente artículo argumenta que la presencia de pandillas y estudiantes relacionados a ellas en los alrededores del centro educativo puede transformar las relaciones educativas y la autoridad docente; al mismo tiempo, provoca un deterioro en la calidad de la educación. En el siguiente apartado, se presentará la metodología empleada en el estudio, seguida por el enfoque conceptual que facilitará la interpretación de los resultados presentados en la cuarta sección. En el apartado final, se profundizará sobre los resultados del estudio y sus implicaciones.

\section{Investigar en contextos inseguros. Metodología}

El tema de la influencia de las pandillas en las escuelas públicas ha sido abordado mayormente desde los medios de comunicación social, muchas veces relatando anécdotas. Además, las redes sociales han contribuido a generar zozobra y morbosidad, creando mitos urbanos sobre el actuar de las pandillas. La investigación social puede ofrecer un aporte más fundamentado y analítico siempre y cuando se logre superar una serie de desafíos.

Estudiar la influencia de las pandillas sobre las relaciones educativas en escuelas públicas es un tema sensible. Requiere un planteamiento metodológico que, por un lado, permita profundizar en el tema, obteniendo información confiable y válida, $\mathrm{y}$, por otro, cuide la seguridad física y emocional de los informantes y del equipo de entrevistadores. Además, estudiar el tema mencionado puede ser confundido con una investigación policial. Por esa razón, es de suma importancia ser transparente con los objetivos del estudio, garantizar la confidencialidad de la información obtenida y proteger el anonimato de los participantes.
El estudio optó por una metodología exploratoria y cualitativa, con un enfoque de teoría fundamentada. La herramienta principal fue la entrevista semiestructurada, la cual facilitó a los informantes el espacio para contar lo que consideraban pertinente $\mathrm{y}$, al mismo tiempo, orientar la conversación con una guía de preguntas para evitar desviarse demasiado de los temas a abordar. Al entrevistar a varios actores educativos de una misma escuela, se permitió obtener diversas miradas sobre un mismo fenómeno.

En el desarrollo de las entrevistas, el aspecto más importante fue proveer un ambiente seguro, donde se podía establecer una relación de confianza y hablar sobre temas que podían ser delicados o difíciles para los informantes. Por el contrario, realizar la entrevista en la misma escuela, con la presencia cercana de pandilleros o alumnos cercanos a ellos, podría generar estrés y miedo en los entrevistados, y perjudicar la calidad de la información. Por esa razón, se escogieron lugares fuera de la institución, con ambientes cómodos, espaciosos y tranquilos. Además, las entrevistas se desarrollaron simultáneamente con los diversos miembros de la comunidad educativa; es decir, con el director, docentes, padres y madres de familia. El objetivo era prevenir que los otros informantes podían escuchar lo que contaba el entrevistado. De esa manera, se creaban las condiciones para proteger la confidencialidad, evitar la filtración de la información y, al mismo tiempo, disminuir la probabilidad de respuestas sesgadas.

La selección de la muestra fue orientada por la necesidad de entender la influencia de las pandillas en las escuelas de manera amplia, no interesaba únicamente las características particulares de un centro educativo. Por esta razón, se buscaron escenarios variados: escuelas rurales y urbanas, ubicadas en distintos departamentos del país, en los linderos de diferentes territorios pandilleriles $y$ en zonas plenamente controladas por una pandilla. Para seleccionar las escuelas, fue esencial encontrar los gatekeepers; es decir, las 
personas o instituciones que podían facilitar el acceso y generar un vínculo que produjera confianza. Entre otros, debían comunicar a los potenciales participantes que el estudio no tenía como propósito evaluar el centro educativo, ni el trabajo del Ministerio de Educación, Ciencia y Tecnología (MINEDUCYT), sino que era una investigación académica que tenía un objetivo más amplio de conocer la situación en la escuela y, además, independiente del MINEDUCYT.

La muestra final fue conformada por seis centros educativos, tres rurales y tres urbanos, de tres departamentos ubicados en las tres zonas del país (occidental, oriental y central). En la tabla 1, se presenta una descripción general de los centros educativos de la muestra.

Tabla 1. Resumen de las características de la muestra de centros educativos

\begin{tabular}{|c|c|c|c|c|l|}
\hline $\begin{array}{c}\text { N. }{ }^{\circ} \text { de } \\
\text { centro } \\
\text { educativo }\end{array}$ & Región & $\begin{array}{c}\text { Ubica- } \\
\text { ción }\end{array}$ & $\begin{array}{c}\text { Matrícula } \\
\text { aproximada }\end{array}$ & $\begin{array}{c}\text { N. }{ }^{\circ} \text { de } \\
\text { docentes }\end{array}$ & Descripción de la situación de seguridad \\
\hline 1 & Occidente & Rural & 300 & 9 & $\begin{array}{l}\text { Controlada por un grupo pandilleril. La ubica- } \\
\text { ción geográfica, cerca de una carretera, provee } \\
\text { seguridad. }\end{array}$ \\
\hline 2 & Occidente & Urbana & 700 & 17 & $\begin{array}{l}\text { Zona céntrica y comercial, en una zona en } \\
\text { disputa entre grupos pandilleriles. }\end{array}$ \\
\hline 3 & Centro & Rural & 1700 & 36 & $\begin{array}{l}\text { Zona neutral, pero cerca de una zona en } \\
\text { disputa entre dos grupos pandilleriles. }\end{array}$ \\
\hline 4 & Centro & Urbana & 700 & 23 & $\begin{array}{l}\text { En una zona controlada por una pandilla, muy } \\
\text { conflictiva. }\end{array}$ \\
\hline 5 & Oriente & Rural & 200 & 7 & $\begin{array}{l}\text { Una zona muy aislada, pero controlada por } \\
\text { un grupo pandilleril. Es necesario cruzar líneas } \\
\text { de control territorial de pandillas para salir del } \\
\text { cantón, por lo que es un lugar muy conflictivo. }\end{array}$ \\
\hline 6 & Oriente & Urbana & 700 & 26 & $\begin{array}{l}\text { Es una zona controlada por una pandilla, pero } \\
\text { tranquila por la presencia de las fuerzas de } \\
\text { seguridad. }\end{array}$ \\
\hline
\end{tabular}

Fuente: entrevistas propias y datos del censo escolar del MINEDUCYT.

Después de las entrevistas, las mismas fueron transcritas y la calidad de las transcripciones revisada. Se realizó el análisis con un total de 52 entrevistas de participantes de seis escuelas. Cada escuela fue analizada como un caso en sí mismo y después los resultados de cada institución fueron comparados con los de las otras; así la investigación consistió en seis estudios de casos. El contenido de las entrevistas fue analizado desde la perspectiva de la teoría fundamentada o grounded theory (Glaser \& Strauss, 1967; Chamaz, 2014). El análisis se basó en el ordenamiento, estructuración y codificación sistemática de los contenidos, para generar categorías conceptuales e interpretaciones teóricas que orientaran las conclusiones. Este proceso fue apoyado por el programa de análisis cualitativo NVivo 10. Los procesos y las dinámicas sociales identificadas -que se desarrollan en las escuelas frente a la presencia de alumnos cercanos a las pandillas- se mencionarán en el apartado de los hallazgos.

Sin duda, las entrevistas semiestructuradas realizadas a los actores educativos de una cantidad reducida de centros educativos públicos no pueden generar resultados representativos para todas las escuelas públicas de El Salvador. No obstante, un estudio de casos 
que toma en cuenta los diferentes escenarios en los cuales las pandillas están presentes e influyen sobre el desempeño de los profesores, su relación con los estudiantes y el funcionamiento cotidiano de los centros educativos puede llegar a un análisis que identifica las dinámicas sociales, relaciones interpersonales y experiencias relacionadas con dicha presencia.

\section{Aproximaciones conceptuales a las relaciones y la calidad educativas}

La educación en sí implica una serie de relaciones, tanto internas como externas, que son esenciales para el cumplimiento de la función de la escuela en el desarrollo personal de los estudiantes y el colectivo, orientándolos hacia una sociedad con mayor cohesión social. Es decir, la educación debe contribuir a "construir comunidades más sanas, productivas, sostenibles, democráticas y con un fuerte tejido social" (FES, 2017, p. 10). Para comprender el marco de esta investigación, es necesario mencionar algunos conceptos y alcances de las relaciones educativas, la relación específica que se comprende como autoridad pedagógica y su influencia en la calidad de la educación.

El funcionamiento cotidiano de la escuela depende de una serie de relaciones sociales entre los actores internos y externos al centro educativo local. La Ley General de Educación (Asamblea Legislativa de El Salvador, 1996), en su artículo 67, define esas relaciones como "comunidad educativa", la cual está conformada por el personal directivo, los docentes, los estudiantes, los padres y las madres de familia. Adicionalmente, el Programa Social Educativo 2009-2014 (MINED, 2009) define una comunidad educativa ampliada, teniendo en cuenta a los actores de la comunidad aledaña (sociales, culturales, económicos y políticos) con un interés activo en la escuela y los procesos educativos (ver también PNUD, 2018).

El presente estudio define relaciones educativas como "las diferentes relaciones en torno del acto educativo, entre los actores internos al centro educativo (p. ej., profesor-alumnos, director-profesor) y actores externos (p. ej., profesor-padres de familia, director-jefe de policía), que forman parte integral del funcionamiento del centro educativo". En este sentido, la relación educativa más reconocida es la del docente con los estudiantes. El acto concreto de educar involucra la confianza, la comunicación, el cuidado mutuo y la convivencia (Touriñán, 2019). La interacción entre el docente y el estudiante de enseñar y aprender, en que constituye la relación pedagógica, muestra que la educación es "una producción de socialización humana" (Sanz Ponce y Serrano Sarmiento, 2017, p. 179), caracterizada por roles definidos, comunicación, convivencia y clima afectivo (positivo o negativo). Esos procesos suceden mayormente en el espacio físico del aula.

Las relaciones educativas son esenciales para la educación que una escuela aspira ofrecer, ya que "la relación intelectual y emocional en el aula debe entenderse como fundamental para contribuir a mejorar la calidad de la educación" (Merino, 2019, p. 181). Las relaciones sostienen los procesos de enseñanza y también el cumplimiento de las metas educativas. Por esa razón, acercarse a la calidad incluye tener en cuenta múltiples componentes educativos como el funcionamiento de la escuela, el trabajo docente y el aprendizaje de los alumnos, no solo las notas (Murillo y Román, 2010).

Un aspecto esencial de la relación educativa es la autoridad pedagógica. Tradicionalmente, está comprendida como la facultad que posee el o la docente para conseguir la atención, motivación y obediencia de los estudiantes (Poblete Zamora y Zerón Rodríguez, 2010). Es una relación dinámica $e$ interactiva basada en "ponerse de acuerdo" para lograr el proceso de enseñanza-aprendizaje. Sin embargo, en la práctica, esa relación entre profesor y alumno no es considerada tan natural. Muchas veces, depende de una autoridad legítima que está basada en el consenso del grupo, en una obligación percibida de obedecer por un contexto social compartido y la existencia de normas comunes (Wrong, 
2002, citado por Macleod et al., 2012). En general, el uso del concepto tiende a ser ambiguo y muchas veces restringido a estrategias para controlar conductas indisciplinadas de los alumnos, pero también incluye la aceptación por parte de los estudiantes de que los docentes tienen mayores conocimientos y pueden aprender de ellos. Representa una autoridad legítima y competente (Macleod et al., 2012).

En situaciones específicas, donde la presencia y control de las pandillas en la comunidad aledaña afecta la seguridad y la conducta de profesores y alumnos en la escuela, la autoridad pedagógica se refleja en la disposición de los alumnos por entablar una relación conducente a la enseñanza y el aprendizaje, a pesar de la atracción de estar en las calles con los pandilleros y las dificultades y los riesgos que enfrentan para llegar y asistir al centro educativo. Por esa razón, en el presente estudio, se define la autoridad pedagógica como la aceptación y la disposición de los estudiantes a recibir la influencia del accionar docente (Abrate et al., 2015). En esencia, es una relación dinámica e interactiva, basada en "ponerse de acuerdo" para realizar un esfuerzo de enseñar y aprender. No obstante, en El Salvador, la existencia de otros poderes cerca de la escuela, especialmente las pandillas, añaden otro elemento de autoridad a la comunidad educativa. La presencia de personas cercanas a la pandilla puede generar un debilitamiento de las relaciones educativas, la autoridad pedagógica y trastocar el desarrollo de los procesos de enseñanza-aprendizaje, tal como se demostrará en el siguiente apartado.

\section{Educar en contextos de pandillas. Los resultados}

Ante la importancia de las relaciones entre los miembros de la comunidad educativa y las consecuencias que un debilitamiento de ellas puede significar para la educación que puede ofrecer la escuela pública, a continuación, se esbozan tres procesos interrelacionados que pueden provocar la presencia de estudiantes relacionados con las pandillas en el entorno escolar: la aparición de temor, la provocación de una nueva forma de autoridad y la disrupción de los procesos educativos. Si son mal manejados, esos pueden tener serias consecuencias para las relaciones educativas en el aula y, consecuentemente, para la calidad de la educación.

El temor en las relaciones educativas. Confianza versus distancia

La presencia de estudiantes vinculados con las pandillas afecta la relación educativa en el momento en que genera ansiedad y temor. De la misma manera que las pandillas establecen el control sobre las comunidades o colonias que consideran sus territorios, especialmente inculcando miedo por el renombre de ser una agrupación violenta y por los actos reales de violencia que demuestran, las relaciones en el centro educativo pueden sentir su presencia. Aunque las pandillas no necesariamente ejecutan actos de violencia en las escuelas -los entrevistados mencionaron pocos o nulos incidentes de violencia física directos-, el miedo presente en la comunidad puede rebalsar a la escuela. Es suficiente que los alumnos o padres de familia hagan insinuaciones o mencionen su relación con miembros de las pandillas para que se activen la ansiedad y el temor en los educadores. Esa ansiedad y ese temor se vuelven parte de la vida cotidiana e impregnan las relaciones con los alumnos. Se crea un ambiente de incertidumbre, ya que muchas veces no saben si esa relación con los pandilleros es real o si ellos cumplirán o no las insinuaciones o amenazas.

La presencia de ansiedad y temor en el centro educativo, esté respaldada o no por hechos concretos, es un impedimento para establecer relaciones de confianza entre profesores y alumnos. La confianza es un aspecto fundamental de la relación educativa y, además, es parte del fundamento de la aceptación y la disposición de los alumnos por aprender. No obstante, las conductas errantes, provocadoras o amenazantes impiden que la confianza en los alumnos se desarrolle porque el docente no confía en que los alumnos 
cumplan su parte del implícito acuerdo mutuo para realizar un esfuerzo interactivo de enseñar y aprender. La confianza en los alumnos se vuelve un elemento sensible de la relación entre profesor y alumno, obligando a tener cierto cuidado y cautela; por ende, los docentes intentan mantener distancia, evitando desarrollar relaciones de confianza con los estudiantes.

La distancia se vuelve parte de una estrategia para protegerse a sí mismos en situaciones difíciles, cuando los profesores tienen temor a los posibles perjuicios que una relación cercana con los alumnos o el hablar de ciertos temas les pueden causar. De esta manera, intentan dar sus clases manteniendo distancia y limitando desarrollar relaciones de confianza con ellos, como las siguientes citas ejemplifican.

"Bueno, lo que nosotros hacemos es, si ellos llegan, nosotros damos la clase normal. Los saludamos y, como le digo, uno lo que tiene es no ser amigos ni enemigos de ellos, pero mantener un margen, no darles la confianza. Porque, a veces, por eso ha fracasado mucha gente. Porque tal vez les dan la confianza y les cuentan cómo viven ellos, cómo es su familia; $y$ de allí lo comienzan a averiguar a uno" (203-V-docf).

"Tengo una buena relación con ellos. Eh..., eso sí, que procuro no..., no..., no darme mucho, porque eso después puede acarrear problemas, ¿verdad? Sobre todo porque yo, como soy de la misma comunidad y me conocen en todo el sector, ¿verdad? Eh..., no puedo extenderme más mi..., dentro de mi..., mi relación de confianza, digamos" (310-III-docm).

"Yo les demuestro confianza, pero con respeto. Vaya, en el sentido de que ellos necesitan de mí y yo necesito de ellos, pero como una relación. Yo les digo: 'Yo lo que yo puedo, yo se los doy a conocer a ustedes y también aprendo de ustedes'. Entonces, genero confianza en ellos, en ese sentido, para poder trabajar" (101-I-dirm).

Las consecuencias del miedo y temor en las relaciones educativas, tanto con la comunidad educativa, como con los estudiantes en el aula, afectan a los docentes de varias maneras. Por una parte, ejercer la profesión docente en un ambiente de incertidumbre, con potenciales y reales amenazas, crea un ambiente de aislamiento e impotencia; por otra, convivir con una constante posibilidad de amenazas a su seguridad personal puede tener consecuencias en la salud mental y física de los docentes. Hay pocas estrategias individuales disponibles para poder continuar el trabajo, las más importantes son: tener paciencia, no mostrar miedo y no complicarse la vida. Muchas veces, las consecuencias incluyen un cierto aislamiento por parte de los docentes hacia los alumnos y la comunidad aledaña.

\section{La aparición de una autoridad coercitiva inversa}

La ansiedad y el temor que sufren los docentes ante la presencia de estudiantes con vínculos con las pandillas producen una modificación en la autoridad pedagógica y abre el espacio al surgimiento de una nueva forma de autoridad, específicamente en el aula. Los alumnos con vínculos con las pandillas generan dinámicas disruptivas que cuestionan las relaciones normadas y socialmente aceptadas, como la autoridad del maestro de organizar e impartir la clase como a él o ella, siendo profesional, le parece más idóneo. Además, influyen en el interés por aprender y la dedicación en el estudio de los estudiantes comunes, es decir, no vinculados.

Los alumnos cercanos a las pandillas - ya sea por tener algún familiar en las pandillas o por relacionarse comúnmente con ellaspueden sentirse en la posición de sortear la autoridad del profesor. Al lucir su relación con las pandillas, estos alumnos se sienten libres de mostrar conductas errantes o, incluso, amenazantes, con el objetivo de imponer sus 
ideas o reglas. Muchas veces, asumen impunemente un rol de un joven indisciplinado $e$ intocable, que hace lo que quiere, especialmente interrumpiendo o impidiendo la clase que el docente desea dar. En el aula, esos alumnos pueden crear dinámicas sociales que van cuestionando y disminuyendo la autoridad del profesor.

A esta nueva forma de relacionarse de los estudiantes en el aula se le puede llamar "autoridad coercitiva inversa". Esta autoridad coercitiva entorpece la relación pedagógica con el docente y se basa principalmente en comportamientos desafiantes, perturbadores o amenazantes por parte de los alumnos que se sienten fortalecidos y respaldados por su relación con la pandilla. Asimismo, se nutre de la influencia que tienen sobre otros estudiantes - el rol de liderazgo que adquieren-y el temor generado en los docentes.

Los mencionados alumnos pretenden que las pandillas respondan por ellos cuando sienten que los tratan mal o les faltan el respeto; es decir, cuando alguien quiere parar sus acciones o que realicen algo que no están dispuestos a hacer. La relación de cercanía con la pandilla puede darse - por ejemplo- por medio de los padres, un hermano, tío, etc., que está vinculado a ella; o, incluso, cuando el alumno mismo anda en presencia de sus miembros. Además, esta relación es conocida por los profesores y los demás alumnos, no existe la necesidad de mencionarla, porque los estudiantes exhiben sus conductas provocadoras y desafían a los profesores. El surgimiento de esa nueva autoridad suele quedar implícito en actitudes insolentes y conductas errantes, pero es entendido perfectamente por los demás.

"Hay unos que interrumpen la clase; se quieren hacer como los chistositos. A lo mejor por el efecto líder..., a lo mejor dicen algo y los demás se ríen. Entonces, como que interrumpen la secuencia didáctica" (101-I-dirm).
"Que él quería poner sus reglas. No quería.... Él no copiaba, no llevaba cuaderno. Pero él quería ir a hacer sus reglas, ir a molestar. Porque sabía que había alguien detrás de él, que lo respaldaba" (110-II-docf).

"No quieren hacer nada. Ellos son los que quieren mandar. Hubo un caso que tuve de ese niño que no quería hacer nada, llegaba a hacer desorden al aula. Solo porque él era marero quería intimidarme" (320-IV-docf).

Las dinámicas generadas por los alumnos cercanos a las pandillas influyen sobre la dedicación al estudio y el interés por aprender de los otros estudiantes. Comunican a los demás la atracción de pasar el tiempo divirtiéndose en el aula, de no obedecer al profesor y hacer desorden. De esa manera, provocan, por un lado, que la autoridad pedagógica que corresponde al docente disminuya $y$, por otro lado, que los procesos de enseñanza y aprendizaje se diluyan.

El desarrollo de una autoridad coercitiva en el aula no solo depende de las interacciones entre alumnos y docente en el aula, sino también de la actitud de la pandilla local frente a la educación. Cuando la pandilla demuestra desinterés en el funcionamiento de la escuela o, al contrario, su interés está asentado en querer controlar lo que pasa dentro, el desempeño educativo de los profesores y el funcionamiento del centro educativo pueden verse gravemente afectado por la conducta de los estudiantes cercanos a ellas.

Al contrario, cuando las pandillas tienen un interés explícito en el buen funcionamiento del centro - por ejemplo, cuando ellos mismos tienen hijos en la escuela o estudiaron allí y tienen aprecio a los profesores - son capaces de llamar la atención a los alumnos cercanos y exigirles cierta disciplina, dedicación a lo enseñado en la escuela y respeto al personal docente. De esa manera, los miembros de las pandillas pueden adquirir una influencia más positiva sobre el rumbo de los procesos educativos en la escuela. En algunos casos, 
incluso, solicitan al director que los llame cuando los alumnos cercanos no hagan caso a su profesor o interrumpan las clases.

No obstante, cuando se da una confrontación entre la autoridad pedagógica y la autoridad coercitiva inversa, la suelen ganar los alumnos relacionados con las pandillas. Las amenazas del uso de la fuerza física, que forman el fundamento de la autoridad coercitiva, no forman parte ni tienen respuestas obvias en el repertorio de la autoridad pedagógica. La amenaza, implícita o explícita, es el rechazo de la influencia del accionar docente - de la relación pedagógica- por parte de los alumnos. Los límites que el surgimiento de la autoridad coercitiva inversa establece a la relación educativa y autoridad pedagógica se vuelven manifiestos cuando, frente a alguna inconformidad o resistencia del profesor, la respuesta del alumno implica una amenaza a su persona. Aunque la eficacia de una autoridad coercitiva se fundamenta sobre la credibilidad de las amenazas con fuerza o violencia, el presunto respaldo o la cercanía del alumno a las pandillas quita muchas dudas sobre la posibilidad de que se realice la amenaza.

\section{Dónde queda la calidad de la educación}

La calidad de la educación que puede ofrecer un centro educativo público en estos contextos se ve disminuida por la ansiedad $y$ el temor entre el personal educativo, el deterioro de las relaciones pedagógicas y el detrimento del ambiente en el aula. El debilitamiento de los procesos educativos perjudica a todos los alumnos; afecta a los estudiantes relacionados con la pandilla, quienes muchas veces no llegan a estudiar, y también a los que se dejan llevar por el ejemplo de ellos.

Por una parte, los alumnos vinculados a las pandillas, además de exhibir acciones errantes o amenazantes, a menudo no llevan cuadernos y tampoco toman notas. De esa manera, demuestran concisamente la falta de interés por lo enseñado. Cuando estos estudiantes causan disrupciones en el aula, los profesores no pueden impartir sus clases de la manera prevista y preparada. Ello afecta aún más los avances de la materia y, en términos más generales, los procesos educativos que la escuela puede desarrollar. Por otra parte, los estudiantes que sí quieren aprender, que esperan avanzar en sus estudios y ambicionan un mejor futuro, ya no están expuestos a procesos educativos acertados y eficaces. Además, los alumnos que constituyen una autoridad coercitiva inversa no solo retan $y$ se imponen ante el personal educativo, sino también ante los demás alumnos. De esta manera, estropean las condiciones necesarias para lograr una calidad educativa deseada. La enseñanza de las materias se atrasa $e$, incluso, algunas partes del programa quedan sin mayor elaboración.

"Cuando uno jornaliza [planifica el desarrollo del programa], uno tiene cierto tiempo para desarrollar determinada unidad, ¿verdad? Entonces, si la clase se ve interrumpida, el tiempo obviamente se alarga y a veces no se logra cumplir el programa" (101-I-dirm).

"Nos atrasamos. Hay cierto límite de atraso porque no se puede avanzar con tantas limitantes. [...] El problema más grave es que la inasistencia de ellos es grande. La otra es que no tienen..., ellos no le ven el interés por estudiar. Eso lo ven como algo: '¿qué? ¿Para qué?, pues. ¿Para qué lo voy a hacer?’” (201-V-dirf).

"Fíjese que esto hace que el rendimiento sea menos, porque hay niños que uno puede como visualizar que les tienen temor. A veces logran dominar al grupo, verdad, y esto hace que los rendimientos bajen en alguna forma; a veces por las tareas. A lo mejor no las hacen por no asistir. Entonces, eso afecta en la educación, afecta bastante" (102-I-docf).

Adicional al abandono de la escuela por parte de alumnos relacionados con las pandillas, a los que no les gusta estudiar, los centros educativos también sufren deserción escolar de estudiantes comunes, quienes abandonan los estudios por razones de ansiedad y temor 
de los pares cercanos a una pandilla o por el lugar de residencia. Muchas de las amenazas dirigidas a alumnos comunes están relacionadas con vivir en un barrio o una colonia donde hay presencia de una pandilla rival a la que está en los alrededores del centro educativo. Cuando los alumnos vinculados o los miembros mismos de las pandillas se dan cuenta de que un alumno reside en una colonia con presencia de sus rivales, rápidamente le hacen saber que no debe acercarse más a la escuela. En otros casos, si tienen que cruzar límites territoriales en el camino a la escuela, existen mayores riesgos que los alumnos abandonen la escuela. En diferentes casos, la matrícula del centro educativo ha sufrido un descenso importante por esas razones. De esa manera, la presencia de las pandillas en los alrededores de los centros educativos públicos puede generar varias dinámicas sociales que provocan una disminución de la integración educativa de los jóvenes.

\section{Discusión y conclusiones}

La realidad de la escuela en situaciones de riesgo, con la presencia de pandilleros en la comunidad aledaña o alumnos relacionados con ellos en el centro educativo, obliga a remirar y reconceptualizar las relaciones educativas, la comunidad educativa, la autoridad pedagógica y el acceso a la educación en un ambiente de inseguridad, temor e incertidumbre. Las metas educativas referentes a la cohesión social y la calidad de la educación son obstaculizadas por la presencia de una fuerza que actúa muchas veces en contra de la labor educativa; es decir, la apariencia de nuevos patrones de autoridad que alteran las relaciones educativas.

Ante la configuración particular de los alumnos relacionados con las pandillas en el aula y su relación con el profesor, es necesario examinar el tema de la autoridad pedagógica. No solo es importante indagar cómo la relación docente-estudiante es afectada, sino también qué tipos de relaciones de autoridad pedagógica parecen ser más efectivos para mantener el trabajo educativo y cumplir el rol docente en el proceso de enseñanza-aprendizaje en los mencionados contextos. En El Salvador, las tensiones sobre la calidad de la educación pública, los recursos y las metodologías utilizadas en el aula son agravadas por el hecho de que en muchos lugares las pandillas se consideran a sí mismas la autoridad local. En esas situaciones, las pandillas definen por sí mismas cómo ver la importancia de las relaciones educativas y la autoridad pedagógica que representa la escuela. Los miembros de las pandillas pueden mantener una actitud de oposición a la autoridad de la escuela y del docente. Pero también pueden aceptar la autoridad pedagógica de los profesores y permitir que funcione el proceso de enseñanza y aprendizaje para que los niños $y$ jóvenes de la zona -que a veces incluyen sus propios hijos - puedan acceder a otras oportunidades.

Ante todo, el temor actualmente experimentado por los docentes es un fenómeno nuevo en el sistema educativo y es importante señalarlo. Las escuelas públicas estaban debilitadas en cuanto a la carencia de recursos, los pobres resultados en la formación docente (Hernández, 2014) y la desmotivación del personal docente por las condiciones de trabajo y salarios (Edwards Jr. et al., 2017). Sin embargo, el hecho de limitar sus acciones y trabajar con temor con actores de la misma comunidad educativa es un reto que nunca habían experimentado. Además de afectar su desempeño en el trabajo, la situación genera un gran conflicto moral, porque la formación docente les inculca cierta vocación sobre sus funciones de educar y orientar a los estudiantes, $y$ hoy no lo pueden hacer.

Otra consideración relevante es que el derecho a la educación se ha convertido en lema y compromiso en las últimas tres décadas en América Latina. Este derecho se ha entendido como acceso a la escuela. Este estudio demuestra que esa definición es insuficiente y que debe ampliarse al derecho de recibir una educación de calidad. La oportunidad de asistir a la escuela del miembro 
de la pandilla, que muchas veces no tiene el objetivo de estudiar ni avanzar en la escuela - más bien desea pasar lo bien un rato o ayudar a controlar la escuela como parte del territorio- violenta el derecho de los estudiantes no vinculados a pandillas de asistir y recibir una educación de calidad. Es decir, los estudiantes comunes tienen el derecho de asistir a la escuela, sentirse seguros y tener las condiciones necesarias para aprender. Una escuela pública ya debilitada desde antes no tiene las posibilidades de atender a los estudiantes vinculados a pandillas y mucho menos de ser una fuerza de rehabilitación o prevención. Quienes se llevan la peor parte son los estudiantes comunes, los no vinculados a las pandillas, los que no pueden llegar a la escuela, los que no se sienten seguros ni pueden avanzar en sus estudios como oportunidad de vida.

Las situaciones anteriormente descritas son difíciles y muchas van más allá de las situaciones para las que los profesores y directores están preparados. La formación docente no los prepara para situaciones de inseguridad y pérdida de autoridad, para ir al trabajo con ansiedad y, además, temiendo las amenazas que los alumnos relacionados con las pandillas o sus familiares pueden expresar. No es de extrañar que la angustia y el temor perjudiquen la salud mental y física de los docentes. Algunos profesores expresan que sufren de tensión vascular o alta presión arterial; otros, que pasan consultas médicas; otros, que buscan apoyo de un psicólogo, una iglesia o personas cercanas para sentirse acompañados, desahogarse de las experiencias en la escuela, encontrar apoyo moral y buscar soluciones. "Lo único que últimamente nos hemos sentido que pasamos estresados, pasamos mal de los nervios, que nos da ansiedad. Nos están desarrollando otras cosas actualmente como maestro" (102-SO1-doc). Tal vez el tema más común expresado en casi en todas las entrevistas es que el MINED desconoce o no sabe manejar las situaciones que ellos viven. Por esa razón, muchos profesores y directores se sienten solos en sus luchas por mantener al centro educativo funcionado $y$ para dar educación de calidad a los jóvenes.

En fin, corresponde repensar las expectativas de qué se debe y qué se puede hacer desde la escuela para contribuir a disminuir la violencia (Novelli \& Lopes Cardozo, 2008). El eje 1 del Plan El Salvador Educado (CONED, 2016) pide a la escuela ser "eje central de la prevención", que en el contexto de presencia de pandillas resulta ser muy ambicioso. Esa pretensión carga a la escuela y a los equipos docentes con una gran responsabilidad frente a entornos sociales que demandan múltiples actividades y requieren mucho presupuesto. Además, la Política de Convivencia Escolar y Cultura de Paz (MINED, 2018b) promueve una convivencia genérica que no reconoce las complejidades de las dinámicas en las comunidades educativas que enfrentan la presencia de pandillas.

La presencia de las pandillas provoca dinámicas específicas en el entorno de la escuela, implica interacciones que pueden ser muy dificultosas e interfieren con el proceso educativo: es un asalto a las relaciones educativas. Se necesita comprender a profundidad esos procesos y relaciones; $y$, además, entender que cada colonia o comunidad tiene sus propias características sociales, relaciones educativas y con la pandilla. Aunque las escuelas públicas en estos contextos necesitan más apoyo y acompañamiento del MINED y la sociedad civil en general, también se debe reconocer que no hay recetas únicas. Solo partiendo de la situación local se pueden tomar decisiones sobre qué hacer, definiendo las prioridades con objetivos claros para crear un ambiente seguro en la escuela sin ansiedad y temor, fortalecer las relaciones educativas - especialmente la relación pedagógica entre docentes y estudiantes-, mejorar la calidad de la educación y así el funcionamiento de la escuela pública.

\section{Referencias bibliográficas}

Abrate, L., Juri, M. A. y Cauteren, A. V. (2015). La autoridad pedagógica en la 
escuela. Decires de los docentes. Cuadernos de Educación, XIII(13), 1-13. https://revistas. unc.edu.ar/index.php/Cuadernos/article/ view/11480/11922

Asamblea Legislativa de El Salvador. (1996). Ley General de Educación.

Baires, S., Martel, R., Romero, C. y Sánchez, C. (2006). Violencia urbana y recuperación de espacios públicos. El caso del AMSS. PNUD y UCA.

Chamaz, K. (2014). Constructing Grounded Theory (2nd. Ed.). Sage Publications Ltd.

Consejo Nacional de Educación [CONED]. (2016). Plan El Salvador Educado. Por el derecho a una educación de calidad. https:// siteal.iiep.unesco.org/sites/default/files/sit accion_files/siteal_el_salvador_0214.pdf

Cruz, J. M. (2007). Street Gangs in Central America. UCA Editores.

Cruz, J. M., Rosen, J., Amaya, L. E. \& Vorobyeva, Y. (2017). The New Face of Street Gangs: The Gang Phenomenon in El Salvador. Florida International University \& FUNDE.

Cuéllar-Marchelli, H. y Góchez, G. (2017). La pertinencia de las estrategias para prevenir la violencia escolar en El Salvador. Serie de investigación 1-2017. FUSADES.

Edwards Jr., D. B., Martin, P. y Flores, I. (2017). Education in El Salvador. Past, present, prospects. En C. M. Posner, Education in Central America and the Latin Caribbean (pp. 141-168). Bloomsbury.

Flores, M. R. y Galdámez, L. (2021). Mapa de intervenciones educativas en centros educativos de zonas con presencia de pandillas. Maestría en Política y Evaluación Educativa, Universidad Centroamericana José Simeón Cañas.

Fundación para la Educación Superior [FES]. (2017). ¿Y si no termino la escuela? La deserción escolar de la juventud salvadoreña entre 15 y 19 años. FES-ESEN.
Glaser, B. \& Strauss, A. (1967). The Discovery of Grounded Theory: Strategies for Qualitative Research. Aldine Publishing Company.

Hernández, J. (2014). Caracterización de los docentes del sistema educativo salvadoreño. Indicadores para conocer el estado de la profesión docente. FUSADES.

Instituto Universitario de Opinión Pública [IUDOP]. (2019). Entender el hoy para pensar en el mañana. Expectativas ciudadanas sobre la economía, la salud, la educación, la seguridad y la justicia. Universidad Centroamericana Jose Simeón Cañas y Seattle International Foundation.

Macleod, G., MacAllister, J. \& Pirrie, A. (2012). Towards a broader understanding of authority in student-teacher relationships. Oxford Review of Education, 38(4), 493-508. https://www.researchgate.net/publication/254326119_Towards_a broader_understanding_of_authörity_in_student-teacherer_relationships

Merino, E. S. (2019). Repensar la relación educativa desde la pedagogía de la alteridad. Teoría de la Educación. Revista Interuniversitaria, 31(2), 177-196. doi:https:// doi.org/10.14201/teri.20271

Ministerio de Educación [MINED]. (2018a). Observatorio MINED 2018 sobre los centros educativos públicos y privados subvencionados de El Salvador. https://www.mined. gob.sv/EstadisticaWeb/observatorio/2018/ OBSERVATORIO\%20SAN\%20SALVADOR. pdf

Ministerio de Educación [MINED]. (2018b). Política Nacional para la Convivencia Escolar y Cultura de Paz. http://www.miportal. edu.sv/wp-content/uploads/recursos/convivencia/Politica/Pol_Nac_Con_2ed.pdf

Ministerio de Educación [MINED]. (2009). Programa Social Educativo 2009-2014 "Vamos a la Escuela". http://cuscatlan. mined.gob.sv/downloads/Institucional/programa-plan-social-educativo-vamos-a-la-escuela. pdf 
Murillo, F. J. y Román, M. (2010). Retos en la evaluación de la calidad de la educación en América Latina. Revista Iberoamericana de Educación, 53, 97-120. https://rieoei.org/ historico/documentos/rie53a05.pdf

Novelli, M. \& Lopes Cardozo, M. (2008). Conflict, education and the global south. New critical directions. International Journal of Educational Development, 28(4), 473-488. doi:10.1016/j.ijedudev.2008.01.004

Programa de las Naciones Unidas para el Desarrollo [PNUD]. (2013). Informe sobre Desarrollo Humano El Salvador 2013. Imaginar un nuevo país. Hacerlo posible. Diagnóstico y propuesta.

Programa de las Naciones Unidas para el Desarrollo [PNUD]. (2018). Informe sobre Desarrollo Humano El Salvador 2018. iSOY JOVEN! ¿Y ahora qué?

Sanz Ponce, R. y Serrano Sarmiento, Á. (2017). ¿La educación cambia? Repensando el sentido y finalidad de una escuela para todos. Teoría de la Educación. Revista Interuniversitaria, 29(2), 167-184. doi:http:// dx.doi.org/10.14201/teoredu292167184

Savenije, W. (2009). Maras y barras. Pandillas y violencia en los barrios marginales de Centroamérica. FLACSO-Programa El Salvador.

Savenije, W. y Andrade-Eekhoff, K. (2003). Conviviendo en la orilla. Exclusión social y violencia en el Área Metropolitana de San Salvador. FLACSO-Programa El Salvador.
Savenije, W. \& Van der Borgh, C. (2015). San Salvador: Violence and Resilience in Gangland - Coping with the Code of the Street. En K. Koonings \& D. Kruijt, Violence and Resilience in Latin American Cities (pp. 90-107). Zed Press.

Smutt, M. y Miranda, L. (1998). El fenómeno de las pandillas en El Salvador. UNICEF y FLACSO-Programa El Salvador.

Touriñán, J. (2019). La relación educativa es un concepto con significado propio que requiere concordancia entre valores y sentimientos en cada interacción. Sophia. Colección de Filosofía de la Educación, 26(1), 223-279. https://www.redalyc.org/jatsRepo/4418/441857903007/html/index.html

USAID-ECCN. (2016). Rapid Education and Risk Analysis El Salvador. Final report. USAID.

Zamora Poblete, G. y Zerón Rodríguez, A. N. (2010). Caracterización y sentido actual de la autoridad pedagógica en escuelas chilenas de sectores de pobreza. Revista Española de Pedagogía, 68(245), 99-116. https://revistadepedagogia.org/wp-content/ uploads/2010/01/245-06.pdf 